\title{
A study on serum hs CRP as a risk predictor and its diagnostic and prognostic significance in acute coronary syndrome
}

\author{
Dr Divakar Kumar ${ }^{1}$, Dr C.B Sharma ${ }^{2}$ \\ ${ }^{1}$ Senior resident, Department of Medicine, RIMS Ranchi \\ ${ }^{2}$ Associate Professor Department of Medicine, RIMS Ranchi
}

\begin{abstract}
There has been an increasing incidence of coronary artery disease in India and a greater Rise is expected in next few years. coronary artery disease in Indians has been found to be more severe and widespread and have been found to be associated with serious complications and increasing mortality. Thus arises the necessity of a tool which act as a risk predictor,can assess the severity and prognosis of acute coronary syndrome also has value in diagnosis and management. The concentration of CRP ( $3 \mathrm{mg} / \mathrm{dl}$ to >200mg/dl ) at which it is detectable in serum is not sensitive for the value required to determine the cardiovascular risk in otherwise healthy person. So modified technique have been developed which can detect hsCRP by hSCRP assay at a range ( $0.2 \mathrm{mg} / \mathrm{l}-20 \mathrm{mg} / \mathrm{l})$ where it can determine cardiovascular risk in otherwise healthy person and have diagnostic and prognostic significance in ACS. hsCRP is an acute phase reactants, whose level are increased proportional to the extent of inflammation. hsCRP is elevated in AMI and levels correlate with total infarct size in AMI and with prognosis and also has role in management. This study is done to measure the hsCRP levels in ACS and to determine its significance for risk prediction and it's diagnostic, management and prognostic significance. We studied 100 patient of acute coronary syndrome ( STEMI and UA/NSTEMI) admitted in department of Medicine RIMS Ranchi with signs and symptoms of acute coronary syndrome during the time period of 1 year on the basis of history, clinical examination, ECG, echocardiography and TMT findings. The serum hsCRP levels were measured at admission by in vitro quantitative method. Patient were also followed up in hospital and also after discharge.There were 75 males and 25 females. Mean age for male was 49.06 years and for female was 60.4 years. STEMI was seen in 52 patients and 48 patient had UA/NSTEMI.hsCRP levels were elevated in 62 patient out of which 46 patients met with complication and among 38 patient in whome CRP were not elevated only 4 patients met with complication.Raised hsCRP levels are markers of adverse outcome. plasmahsCRP levels on admission serves to identify high risk patients in the settings of ACS. The effective list stratification provided maybe of the specific value for early therapeutic decision making and patient treatment in patients presenting with ACS.hSCRP levels were high in STEMI patients in comparison to UA/NSTEMI and also high hsCRP level were associated with poor prognosis.
\end{abstract}

Keywords: FNAC, USG, HPE, Thyroid Nodule.

\section{Introduction}

Coronary artery disease has been emerged as the major cardiovascular disease of the era and also among the commonest cause of premature death. Developing countries now experience a much greater burden of cardiovascular disease than developed countries. In addition developing countries like India are expected to experience the greatest risk in cardiovascular disease burden in coming few years. The prediction of Indians to coronary artery disease has been confirmed beyond doubt .Indian population are more prone to develop coronary artery disease at younger age. In large part this increase can be explained on the basis of major ongoing sociodemographic changes in developing countries and associated effects on the number of individuals at risk and the levels of cardiovascular risk factors. CAD among Indians has been found to be associated with serious complications and increased mortality at an younger age. the classicaly defined risk factors for atherosclerotic arterial disease are ,dyslipidemia, hypertension ,Diabetes mellitus and cigarette smoking.In ethnic Asian Indians the insulin resistance syndrome, metabolic syndrome, lipoprotein (a), atherogenic dyslipidemic phenotype and some newer emerging risk factors( homocystein, tPA, fibrinogen, factor VII, infections and inflammation) may be more relevant.An underlying genetic susceptibility associated with a modest abnormality in lipid and Lifestyle factor make coronary artery disease assume a malignant course in Asian Indians. From 1960s to 1990s the coronary artery disease prevalence increased two folds (from $2 \%$ to $4 \%$ ) in rural India and three to four folds (3.45\% to $9.45 \%)$ in urban India. Atherosclerotic plaque disruption, coronary thrombosis and vasospasm have been implicated in pathophysiology of acute coronary syndrome since long period. Despite progress in the prevention of cardiovascular disease a significant proportion of first cardiovascular event occur among individuals without traditional risk factor described above.Cardiovascular event develop unpredictably in patient with wide degree of atherosclerotic disease.Thus major area of interest is the study of factor that provoke ischemic event.atherosclerosis of coronary vessels commonly cause ischaemic 
heart disease.The past decade has witnessed an increased recognition that inflammatory mechanism play a central role in pathogenesis of atherosclerosis and its complication.Recently attention has been focused on the potential role of plasma markers of inflammation specially hsCRP as a risk predictor among those at risk for cardiovascular event.With inflammation there will be release of an inflammatory cytokines from the inflamed tissue which stimulates liver to synthesise a number of acute phase proteins including $\mathrm{C}$ reactive protein.In clinical studies it is shown that circulating level of hsCRP were found to correlate with total infarct size in acute myocardial Infarction and with prognosis. Thus hsCRP is an indicator of underlying coronary inflammation as well as extent of myocardial necrosis

\section{Objectives}

1. To study the significance of serum hsCRP as a novel and promising risk predictor among patients of acute coronary syndrome.

2. To study the impact on diagnosis and effective management based on serum hsCRP estimation.

3. To study the prognostic significance of serum hsCRP in acute coronary syndrome patients in relation to morbidity and mortality.

\section{Materials And Methodology}

In the present study 100 cases of acute coronary syndrome were taken from those admitted in department of Medicine RIMS Ranchi with symptoms and signs of acute coronary syndrome during the time period of one year on the basis of history,clinical examination, ECG, 2D echo and TMT finding. On an average 2 cases of acute coronary syndrome w ere admitted per week and it comes to about 100 cases in an year.inclusion criteria for patient is more than 18 years males and female, ST elevation acute MI and NSTEMI and unstable angina.Other patient having elevated hsCRP were excluded.Outcome of this study was, improved without complication and developed complication which affected prognosis like cardiogenic shock, LVF, LBBB ,AV block, V T and VF, SVT, CCF, postinfarct angina, recurrent angina and death.Data obtained was statistical analysed using frequency distribution, descriptive analysis ,student $t$ test, ANOVA, Chi square test to see the association between variables and accordingly inference and conclusion was drawn. The hsCRP was measured by hsCRP essay which is an in vitro quantitative determination of hsCRP in human serum and plasma and this essay is based on latex enhanced turbidimetric immunoassay method. The hscrp essay has a linear range of 0.2 to $20 \mathrm{mg} / \mathrm{dl}$ that extends below the measurement of most conventional CRP assay.

\section{Results}

Total of 100 patients were taken for the study out of which 75 patients were males and 25 patients were female. Maximum incidence of acute coronary syndrome was observed in age group between 40 - 49 yrs accounting for $29 \%$ of patients. Minimum incidence of ACS was noted in $20-29$ years of age group accounting for $2 \%$ of patients. Mean age of incidence of ACS in male was 49.06 years and that of female it was 60.4 years,over all the mean age of occurrence of ACS for all patient was 51.53 years. Males were most commonly involved in both STEMI and UA/NSTEMI patients. Most common presenting complaint was chest pain in $83 \%$ of cases followed by sweating $(72 \%)$, breathlessness $(38 \%)$, vomiting $(11 \%)$ epigastric discomfort $(6 \%)$ ,giddiness (4\%) and diarrhoea and back pain (2\%). Most common risk factor in this study was smoking in $64 \%$ of cases followed by dyslipidemia (46\%), hypertension (32\%), diabetes (22\%), age( $10 \%$ ) and family history of IHD (7\%). In the present study out of 100 patients studied 52 had STEMI among which distribution was as follows:- Extensive anterior wall MI in 24 patient (46\%), Inferior wall MI in 12 (23\%)patients, Anteroseptal wall MI in 7 (13\%)patient ,Anterolateral wall MI in 4 (8\%)patients, Anterior wall with inferior wall MI in 2(4\%)patients, Inferior wall MI with right ventricular extension in 2 (4\%) patient and Inferior wall MI with posterior wall MI the least common type in $1(2 \%)$ of patients. The study showed patient having high hsCRP level who had undergone thrombolytic therapy had high survival percentage in comparison to patient of STEMI who were not thrombolysed but had mean hsCRP level lower than those thrombolysed. Chi square value was 4.29 and 1 degree of freedom ,P value was significant $(\mathrm{P}=0.03)$ which signifies that elevated hsCRP level may have a supportive role in guiding the management of acute coronary syndrome by taking early aggressive measures.

Table - I: Serum hsCRP level in patients with STEMI and UA/NSTEMI

\begin{tabular}{|c|c|c|c|c|c|c|}
\hline \multirow{2}{*}{$\begin{array}{c}\text { hsCRP } \\
\text { level }\end{array}$} & \multicolumn{2}{|c|}{ STEMI } & \multicolumn{3}{c|}{ UA/NSTEMI } & $\%$ \\
\cline { 2 - 7 } & NO & $\%$ & NO & NO & $\%$ \\
\hline$>1 \mathrm{mg} / \mathrm{L}$ & 42 & 68 & 26 & 32 & 62 & 62 \\
\hline$<1 \mathrm{mg} / \mathrm{L}$ & 10 & 26 & 28 & 74 & 38 & 38 \\
\hline Total & 52 & 52 & 48 & 48 & 100 & 100 \\
\hline
\end{tabular}

The present study showed hsCRP level >L was present in $62(62 \%)$ of patients while hsCRP level $<1 \mathrm{mg} / \mathrm{L}$ was present in $38(38 \%)$ of patients. This data shows if patient have high hsCRP levels $(>1 \mathrm{mg} / \mathrm{L})$ 
patient is more likely to have STEMI instead of UA/NSTEMI $(\mathrm{P}<0.001)$ which suggest hsCRP level maybe more sensitive as a supportive marker to other diagnostic tools in diagnosis of STEMI in comparison to UA/NSTEMI, providing its diagnostic value. The cut off value of hsCRP of $1 \mathrm{mg} / \mathrm{L}$ was as per recommendation of CDC/AHA Jupiter trial.

Table - II: Mean hsCRP level and its relationship with mortality in STEMI and UA/NSTEMI patients

\begin{tabular}{|c|c|c|c|c|}
\hline Type of MI & $\begin{array}{c}\text { Mean } \pm \text { SD } \\
\text { hsCRP level }\end{array}$ & No of patients & \multicolumn{2}{|c|}{ Death } \\
\cline { 4 - 5 } & $4.06 \pm 2.24$ & 52 & NO & $\%$ \\
\hline STEMI & $1.8 \pm 1.88$ & 48 & 8 & 46 \\
\hline UA/NSTEMI & \multicolumn{2}{|c|}{} \\
\hline
\end{tabular}

The study showed STEMI patients has higher hsCRP level and higher mortality, which is associated with higher hsCRP level.

Table - III: Serum hsCRP level in patients with and without complications of ACS cases under study

\begin{tabular}{|c|c|c|c|c|c|c|}
\hline \multirow{2}{*}{ HsCRP } & \multicolumn{2}{|l|}{ ACS with complications } & \multicolumn{2}{c|}{$\begin{array}{c}\text { ACS without } \\
\text { complications }\end{array}$} & \multicolumn{2}{|c|}{ al } \\
\cline { 2 - 7 } & NO & $\%$ & NO & $\%$ & NO & $\%$ \\
\hline$<1 \mathrm{mg} / \mathrm{dl}$ & 4 & 11 & 34 & 89 & 38 & 38 \\
\hline$>1 \mathrm{mg} / \mathrm{dl}$ & 46 & 74 & 16 & 26 & 62 & 62 \\
\hline Total & 50 & 50 & 50 & 50 & 100 & 100 \\
\hline
\end{tabular}

This indicates that the patients with high hsCRP level at admission are prone to develop different complications of ACS signifying its risk predictor and prognostic value in developing complications(risk). The Chi square value was 38.2 and at one degree of freedom, $\mathrm{P}$ value was highly significant $(\mathrm{P}<0.001)$.

In the present study mean hsCRP level was highest in patients who developed cardiogenic shock.Maximum percentage $(80 \%)$ of mortality occurred in patients who developed CCF, VT, CHB. In the present study most common complication was LVF followed by cardiogenic shock, CCF,CHB,SVT,VT,PIA,RA,LBBB, while the least common complication was AV block.Patients who dwveloped complication had high hsCRP value $(>1 \mathrm{mb} / \mathrm{L})$. Signifying its prognostic value in terms of morbidity and mortality.

Table - IV: Serum hsCRP level in patients of ACS under study who developed complication

\begin{tabular}{|c|c|c|c|}
\hline \multirow{2}{*}{ HsCRP } & \multirow{2}{*}{$\begin{array}{c}\text { Total no of } \\
\text { patients }\end{array}$} & \multicolumn{2}{|c|}{ ACS patients who developed complications } \\
\cline { 3 - 4 } & 38 & NO & $\%$ \\
\hline$<1 \mathrm{mg} / \mathrm{L}$ & 62 & 4 & 10.52 \\
\hline$>1 \mathrm{mg} / \mathrm{L}$ & 62 & 46 & 74.19 \\
\hline
\end{tabular}

Table - V: Serum hsCRP level in patients of ACS under study who died

\begin{tabular}{|c|c|c|c|}
\hline \multirow{2}{*}{ hsCRP } & Total no of patients & \multicolumn{2}{|c|}{ ACS patients who died } \\
\cline { 3 - 4 } & & NO & $\%$ \\
\hline$<1 \mathrm{mg} / \mathrm{L}$ & 38 & 4 & 10.52 \\
\hline$>1 \mathrm{mg} / \mathrm{L}$ & 62 & 46 & 74.19 \\
\hline
\end{tabular}

The above both tables showed that hsCRP level $>1 \mathrm{mg} / \mathrm{L}$ has value for risk prediction for outcome in ACS patients and have prognostic value in terms of morbidity and mortality and have highly significant $\mathrm{P}$ value $(<0.001)$ at one degree of freedom.

Table - VI: Serum hsCRP level in cases of ACS under study who developed complications, who did not develop complications, who died and who survived.

\begin{tabular}{|c|c|c|}
\hline Cases under study & Mean \pm SD hsCRP levels & No of patients \\
\hline ACS with complications & $4.08 \pm 2.49$ & 50 \\
\hline ACS without complications & $1.80 \pm 1.55$ & 50 \\
\hline Cases under study & Mean \pm SD hsCRP levels & No of patients \\
\hline Patients who died & $5 \pm 2.39$ & 32 \\
\hline Patients who survived & $1.85 \pm 1.5$ & 68 \\
\hline
\end{tabular}

These tables again showed that higher hsCRPlevelsis associated with more complications and death signifying the value of hsCRP as risk predictor and prognostic value in terms of morbidity and mortality.Other investigations like ESR was raised in $62 \%$ of patients, Tlc was raised in $24 \%$ of patients, and Random blood glucose level was raised in $32 \%$ of patients. 


\section{Discussion}

In the present study role of serum hsCRP in Acute coronary syndrome has been thoroughly worked and observed and its significance has been found in terms of risk prediction diagnostic and prognostic significance. The maximum incidence of acute coronary syndrome was seen in age group between 40 to 49 years while in studies by Foussas et al and Suleiman et al mean it was 7th decade. In Indian subcontinent the coronary artery disease occurs decade earlier then the Western population. So mean age of our patient is comparable to above mentioned studies. Sex ratio is comparable to study by Foussas at el and Mohammed Suleiman et al. most common presenting symptom of chest pain is compatible to study by Huggins at el. Most common risk factor in this study that is smoking is comparable to the study by Foussas et al and Suleiman at el and Salim yusuf et al. In our study $52 \%$ patient had STEMI which is comparable to study by Mohammed Suleiman at el. In this study $15 \%$ patient were thrombolysed while in study by Suleiman et al $32 \%$ of patients were thrombolysed. the less number of thrombolysis done in present study was because of late presentation in emergency that is after 12 hour and formed Q wave in ECG. ESR was raised in similar number of patients as was study by Harsberg et al. Total leucocyte count was raised in similar number of patients as that by Thomson et al. In the present study the number of patient who had hsCRP level $>1 \mathrm{mg} / \mathrm{L}$ was $62(62 \%)$. Among them 42(68\%) had STEMI and $20(32 \%)$ had UA/NSTEMI. The number of patients who had hsCRP $<1 \mathrm{mg} / \mathrm{L}$ was $38(38 \%)$. Among them $10(26 \%)$ had STEMI while $28(74 \%)$ have NSTEMI.

these data suggest that if hsCRP level is high $(>1 \mathrm{mg} / \mathrm{L})$ patient is more likely to have STEMI instead of UA/NSTEMI $(\mathrm{P}<0.001)$ which suggest hsCRP levels may be sensitive as a supportive marker to other diagnostic tools in diagnosis of STEMI in comparison to UA/NSTEMI providing its diagnostic value. Similar results were found in studies by SS habib,dystefano R et al and Yildiz Hasan. In the present study mean value of hSCRP in STEMI and UA/NSTEMI patient was 4.06 and 1.8 respectively compatible to study by Distefano et al and Yildiz Hassan et al. Death was also high in STEMI patient corresponding to high hsCRP value. More complication associated with high hsCRP value $(>1 \mathrm{mg} / \mathrm{L})$ was comparable to study by Foussas et al and Suleiman et al which signified its prognostic significance. Jupiter trial showed mean hsCRP level $>1 \mathrm{mg} / \mathrm{L}$ in patient who developed complication which is comparable to present study signifying its prognostic significance. Most common complication of left ventricular failure was similar to that of study by Mohammad Suleiman et al. In the present study more patient died with hsCRP level $>1 \mathrm{mg} / \mathrm{L}$ which is comparable to study by Mohammed Suleiman et al signifying its prognostic value in terms of mortality.

\section{Conclusion}

Coronary artery disease is common in middle and enderly age group. Smoking, diabetes ,hypertension and dyslipidemia are common risk factors. the most common presenting symptoms are chest pain, sweating, breathlessness and epigastric discomfort. Serum hsCRP level is an important biochemical markers for various aspect of ACS and also a very promising novel biochemical marker as a risk predictor for adverse cardiovascular event. Serum hsCRP levels at admission serves to identify high risk patient in setting of acute coronary syndrome. The effective risk stratification provided maybe of specific value for early therapeutic decision making and management of patient who presents with acute coronary syndrome. The raised serum hsCRP level are more sensitive in diagnosis of STEMI in comparison to UA/NSTEMI as a supportive marker to other diagnostic tools, as serum hsCRP levels has been significantly higher in STEMI patients. In patient of ACS with increased serum hsCRP level at admission have been associated with poor prognosis in in terms of mortality and morbidity. This study is not the final verdict. More future studies will either corroborate or contradict this study.

\section{References}

[1]. FOUSSAS SG, Zairis MN, Katsaros et al. Early prognostic usefulness of C reactive protein added to thrombolysis in myocardial infarction risk score in acute coronary syndrome.Aug 2005;96;P533-537

[2]. Suleiman, AronsonD, LEVY et alAdmission C reactive protein levels and 30 day mortality in patients with acute myocardial infarction ,Dec 2003,115;9 p695-701

[3]. Distefavo R , CarpiA. Inflammatory markers and cardiac function in acute coronary syndrome. Differernce in STEMI and in NSTEMImodels 2009 Dec: $773-80$

[4]. Habib SS, Kurdi MI. hsCRP levels are higher in patients with ST elevation than non ST elevevation acute coronary syndrome 2011 Jan;96(1)

[5]. Hersberg PI, Hematocrit and prognosis in patient with acute myocardial infarction, JAMA $1973 ; 219 ; ; 855$

[6]. Thomson SP, GibbonsRJ, Incremental value of the leucocyte differentisl and rapid creatine kinase MB isoenzymes for the early diagnosis of myocardial infarction;1995;122;335

[7]. Yildiz Hassan , IPGMJ vol.12,sup.2013.High sensitive C-Reactive protein levels in patients with acute coronary syndrome.

[8]. Harrisons's principle of Internal Medicine, (19th edition volume 2)

[9]. Paul M Ridker, Jupiter trail2009:2:279-285

[10]. Rifai N, RidkerPM.High sensitivity C - Reactive protein: a novel and promising marker of coronary heart disease. 